\title{
EFECTO DEL ALOJAMIENTO, REUSO DE LA CAMA Y ALMACENAMIENTO EN LA COMPOSICIÓN QUÍMICA DE LA POLLINAZA ${ }^{1}$
}

\author{
Rebeca Zamora-Sanabria ${ }^{2 / *}$, Juan Ignacio Herrera-Muñoz,*, Sebastián Dorado-Montenegro***, \\ Alejandro Saborío-Montero ${ }^{* * * * *}$
}

Palabras clave: Pollinaza; alojamiento; nutrición; pollos de engorde; rumiantes; almacenamiento.

Keywords: Poultry litter; housing; nutrition; broilers; ruminants; storage.

Recibido: $23 / 10 / 18$

RESUMEN

Se efectuaron análisis químicos a 26 muestras de pollinaza fresca obtenida de 18 granjas de pollo de engorde con galeras abiertas o convencionales y galeras cerradas o tipo túnel con camas de un solo uso y reutilizadas de cascarilla de arroz, con el objetivo de evaluar la composición química de la pollinaza, el efecto del tipo de alojamiento y de la reutilización de la cama sobre la composición nutricional. Adicionalmente, 17 muestras fueron almacenadas bajo condiciones ambientales no controladas por 60 días, para determinar el efecto del almacenaje sobre el contenido nutricional. Se realizaron análisis de materia seca a $135^{\circ} \mathrm{C}(\mathrm{MS})$, proteína cruda (PC), fibra detergente neutra (FDN), fibra detergente ácida (FDA), nitrógeno ligado a la fibra detergente neutra (FDN-N), nitrógeno ligado a la fibra a detergente ácida (FDA-N), extracto etéreo (EE), energía digestible (ED), nitrógeno no proteico (NNP), cenizas, lignina (LDA), proteína equivalente $(\mathrm{PEq})$, contenido de calcio y fósforo. El tipo de alojamiento no presentó efecto

1 Este proyecto fue financiado por el fondo de estímulo de la Vicerrectoría de Investigación de la Universidad de Costa Rica. Proyecto 739-B5-088.

2 Autora para correspondencia. Correo electrónico: rebeca.zamora@ucr.ac.cr

Universidad de Costa Rica, Escuela de Zootecnia, Centro de Investigación en Nutrición Animal, Costa Rica. (iD) 0000-0002-9679-4647.

Aceptado: 12/02/19

\section{Effect of housing, recycling and storage} on poultry litter chemical composition. Chemical analysis were performed on 26 samples of fresh single-used and recycled rice husk poultry litter from conventional and tunnel-ventilated housing systems with the objective to characterize its nutrient composition and to study the effect of housing design and litter reutilization on nutritient concentration. In addition, 17 samples were stored under non-controlled environmental conditions for 60 days, in order to determine the effect of storage time on nutritional composition. Samples were analyzed for dry matter (DM) at $135^{\circ} \mathrm{C}$, crude protein $(\mathrm{CP})$, neutral detergent fiber (NDF), acid detergent fiber (ADF), neutral detergent insoluble nitrgogen (NDF-N), acid detergent insoluble nitrogen (ADF-N), ether extract (EE), digestible energy (DE), non-protein nitrogen (NPN), ashes, lignin (LDF), protein equivalent $(\mathrm{PEq})$, and $\mathrm{Ca}$ and $\mathrm{P}$ content. Poultry housing design had no effect on litter nutritional composition; however, recycled bedding showed

\footnotetext{
** Universidad de Costa Rica, Escuela de Zootecnia, Centro de Investigación en Nutrición Animal, Costa Rica. (D) 0000-0001-5004-0826.

*** Universidad de Costa Rica, Escuela de Zootecnia, Centro de Investigación en Nutrición Animal, Costa Rica. (D) 0000-0002-9220-6318.

**** Universidad de Costa Rica, Escuela de Zootecnia, Centro de Investigación en Nutrición Animal, Costa Rica. (DD) 0000-0002-9840-0058.
} 
significativo sobre la composición nutricional de la pollinaza, el material obtenido de las camas reutilizadas en más de 2 ciclos de producción, presentó mayores $(\mathrm{p}<0,05)$ concentraciones de PC, FDA, calcio y fósforo, al compararlo con las camas de un solo uso; esta última presentó menores concentraciones $(\mathrm{p}<0,05)$ de FC y FDN. El almacenaje de la pollinaza presentó un efecto significativo $(\mathrm{p}<0,05)$ en los valores de PC, NNP, EE, FDN, FDA y en la materia seca. La reutilización de la cama, así como el almacenaje de la misma, afectó la composición química de la pollinaza; no así, el tipo de alojamiento. La composición nutricional de la pollinaza analizada permite considerarla como un buen ingrediente para la alimentación de rumiantes, siempre y cuando se acaten las disposiciones del Servicio Nacional de Salud Animal (SENASA) y de otras instituciones gubernamentales e internacionales que regulan su uso y manejo.

\section{INTRODUCCIÓN}

En Costa Rica, la industria avícola se presenta como una de las actividades agropecuarias consolidadas e importantes (Bolan et al. 2010), y que más cambios muestra en sus sistemas de producción (INEC 2017). El PNSA 2019 reporta la existencia de 741 granjas industriales debidamente inscritas ante el Servicio Nacional de Salud Animal (SENASA), de las cuales 282 se dedican al engorde de pollos en instalaciones altamente tecnificadas y especializadas en la producción intensiva. La producción tecnificada incluye el 94\% del total de aves del país (INEC 2017). El INEC 2014 reporta una población de 8339940 pollos de engorde, lo que representa el $47 \%$ de la población nacional de aves domésticas. Las granjas se ubican principalmente en la Región Central (70\%), el 26\% se encuentra en la Región Huetar Norte y la Región Pacífico Central (INEC 2017). higher values $(\mathrm{p}<0.05)$ for $\mathrm{CP}, \mathrm{ADF}, \mathrm{Ca}$ and $\mathrm{P}$, when compared with single-used litter; the latter had lower $(\mathrm{p}<0.05) \mathrm{DM}$ and NDF content. Furthermore, storage time had a significant effect $(p<0.05)$ on CP, NPN, EE, NFE, NDF, ADF and DM. Recycling and storage time of broiler litter affected its nutritional composition; while housing design had no effect. Evaluated poultry litter in this investigation is consider as an appropriate ingredient for ruminant feeding, as long as its usage follows government laws and recommendations from the National Service of Animal Health (SENASA) and other international authorities that regulates its proper use.

La tecnificación avícola incluye el uso de instalaciones abiertas y cerradas, completamente automatizadas, con control ambiental y ventilación mecánica, comederos y bebederos automáticos, con piso de concreto cubierto con diferente tipo y cantidad de cama, y sistemas automatizados para el control remoto de las condiciones internas de las galeras, condiciones que permiten alojar una gran cantidad de aves en espacios menores (Mesa et al. 2017) y producir de forma confortable (Glatz y Pym 2019). Según datos del INEC (2017), de 1984 al 2014 hubo una reducción del 37\% en el número de explotaciones avícolas, pero con un aumento de $257 \%$ en el número de aves. La cantidad de pollinaza que produce cada pollo de engorde depende del tipo material de la cama, del manejo (Bolan et al. 2010), de las instalaciones (Tasistro et al. 2004), del tipo de alimento, de su digestibilidad (Bolan et al. 2010) y de la cantidad de alimento que las aves desperdician durante su alimentación (Leytem et al. 
2007). Un pollo de engorde produce aproximadamente $6,9 \mathrm{~kg}$ de pollinaza por cada $1000 \mathrm{~kg}$ de peso vivo por día (Bolan et al. 2010).

Como consecuencia de esta situación, la pollinaza es el remanente que se produce en mayor volumen y constituye el mayor reto en la producción de pollo de engorde. Este material precisa de un adecuado tratamiento y manejo, (Decreto No. 29285-MAG-S-2001) con el fin de no causar daños al ambiente y contribuir con la sostenibilidad de la producción avícola. El tratamiento más utilizado en Costa Rica es el calentamiento espontáneo por 3 días. El manejo y disposición de las cantidades de pollinaza producidas, sin tratamiento o transformación, podría representar un problema logístico y ambiental de grandes proporciones (Bolan et al. 2010).

A nivel nacional existe poca información para la persona usuaria final de la pollinaza, en relación con su composición nutricional, mineral y química. Estos datos facilitarían tomar decisiones en cuanto al nivel óptimo de inclusión del material en una dieta para rumiantes, el nivel máximo de aplicación como abono orgánico en un cultivo o para definir el método de tratamiento que más se ajusta a las condiciones de cada productor (Cayci et al. 2017, Magdy 2017). En el pasado se realizaron esfuerzos para proveer esta información, como los trabajos presentados por Tobía y Vargas (2000a), Tobía y Vargas (2000b). Sin embargo, se requiere investigación continua, debido a los diversos factores que afectan la composición de la pollinaza.

En los últimos años las camas de cascarilla de arroz se reutilizan frecuentemente, debido a la poca disponibilidad de este material y a la especulación en los precios. La práctica de reutilizar la cama también se realiza para bajar los costos de producción de la carne de pollo (Vejarano et al. 2008, Paganini et al. 2004). Los productores buscan reducir el tiempo de alistado y preparación de las instalaciones entre ciclos productivos y reutilizar el material de la cama cuando el precio de venta de la pollinaza disminuye, debido a la menor demanda como alimento para rumiantes, en algunas épocas del año (Roothaert y Matthewman 1992). Además, la reutilización de la cama se considera una opción para disminuir la generación de residuos y la contaminación ambiental (Tobía y Vargas 2000a).

El uso indiscriminado de la pollinaza, como insumo en la alimentación de rumiantes, sin un tratamiento previo o sin acatar la legislación nacional e internacional, puede ocasionar problemas sanitarios, inclusive causar la muerte de los animales (Garriz y López 2002). Un inadecuado balance de la dieta causa disminución en la producción, pues la pollinaza es un material muy variable en su composición de nutrientes y en su contenido de humedad (Van Rissen 2001, Tasistro et al. 2004). Los animales pueden sufrir intoxicación por exceso de algunos componentes, como cobre $(\mathrm{Cu})$ (Tokarnia et al. 2000, Bolan et al. 2010), y nitrógeno no proteico (NNP) (Roothaert y Matthewman 1992, Staton y Whittier 2006, Galvis et al. 2011). La presencia de micotoxinas, residuos de antibióticos y bacterias como Salmonella, Clostridium y Campylobacter tambien podrían afectar la salud de los rumiantes que la consumen (Van Ryssen 2001, Magdy 2017). Por estas razones, es indispensable conocer con mayor precisión, la composición de este material. El objetivo de este trabajo fue evaluar la composición nutricional de la pollinaza, el efecto del tipo de alojamiento de los pollos de engorde, la reutilización de la cama y del almacenamiento.

\section{MATERIALES Y MÉTODOS}

Para este estudio se analizaron 26 muestras de pollinaza de 18 granjas de pollo de engorde de la línea genética Cobb 500 (Cuadro 1). Todas las granjas presentaron diferente cantidad de galeras, desde 2 hasta 10 galeras de dimensiones similares. Las granjas se ubicaron en los cantones de Naranjo, Palmares, San Ramón, Alajuela y San Carlos, zonas donde se produce la mayor cantidad de pollo de engorde del país (INEC 2017). La extracción de las muestras se realizó durante abril, junio, julio, agosto y noviembre del 2016, enero, marzo, mayo, setiembre y noviembre del 2017. 
Cuadro 1. Cantidad y tipo de muestras analizadas según el tipo de alojamiento.

\begin{tabular}{lc}
\hline Tipo de alojamiento & Cantidad de muestras frescas \\
\hline Galeras abiertas & 14 \\
Galeras cerradas & 12 \\
Total de granjas & 18 \\
Total de galeras o muestras & 26 \\
\cline { 2 - 2 } & Cantidad de muestras almacenadas \\
Galeras abiertas & 8 \\
Galeras cerradas & 9 \\
Total de granjas & 13 \\
Total de galeras o muestras & 17 \\
\hline
\end{tabular}

Algunas de las granjas seleccionadas contaban con los 2 tipos de alojamiento o instalaciones: galeras convencionales o abiertas y cerradas o tipo túnel, por lo que en estas granjas, se recolectaron muestras de los 2 tipos de alojamiento.
En otras granjas los productores reutilizaron la cama en algunas galeras durante el mismo ciclo productivo, por lo que en estos casos, se tomaron muestras de cama reutilizada y sin reutilizar de diferentes galeras de la misma granja (Cuadro 2).

Cuadro 2. Cantidad y tipo de muestras analizadas según el uso de la cama.

\begin{tabular}{lc}
\hline Uso de la cama & Cantidad de muestras frescas \\
\hline Un solo uso & 16 \\
Reutilizadas & 10 \\
Total de muestras o galeras & 26 \\
Total de granjas & 18 \\
\cline { 2 - 2 } & Cantidad de muestras almacenadas \\
\hline Un solo uso & 11 \\
Reutilizada & 6 \\
Total de muestras o galeras & 17 \\
Total de granjas & 13 \\
\hline
\end{tabular}

Todas las galeras utilizaron cascarilla de arroz como material de cama sobre piso de concreto. La mayoría de las galeras tanto abiertas como cerradas estaban construidas con madera y techo de metal, con dimensiones entre $12-16$ $\mathrm{m}$ de ancho y 100 - $150 \mathrm{~m}$ de largo. Las galeras abiertas realizaban ventilación natural mediante el uso de cortinas móviles y ventiladores dentro de las instalaciones. Las galeras cerradas eran ventiladas mecánicamente, a través de entradas 
de aire reguladas y de extractores de aire con control automático mediante sensores. En ambos tipos de galeras se utilizaban comederos y bebederos automáticos.

Para lograr una adecuada descripción y caracterización de las muestras de pollinaza, en cada granja se recolectó información productiva referente a la permanencia de los pollos en la granja, carga animal o densidad, uso de la pollinaza, tipo de piso, reutilización de la cama, tipo de alojamiento, tipo y cantidad de cama, número de ciclos productivos al año, proveedor de alimento balanceado y tamaño de las instalaciones.

Para la obtención de las muestras de pollinaza, se coordinó con los productores para estimar la duración del ciclo productivo y las fechas programadas para el envío de los pollos a la planta de cosecha. Esto permitió tomar las muestras de pollinaza 3 y 4 días antes de la salida de los pollos a la planta de sacrificio.

Se utilizó una muestra por galera. Las galeras se subdividieron en 3 secciones (frente, medio y fondo) y se tomaron 3 sub-muestras, una en cada sección de la galera, al considerar el área de recibo de los pollitos, las entradas de aire, la zona de bebederos y comederos, y la zona de extractores o ventiladores, según el tipo de galera y de acuerdo con lo recomendado por Tasistro et al. (2004).

Para la toma de cada sub-muestra se utilizó un marco de $50 \mathrm{~cm}$ de lado (área de $0,25 \mathrm{~m}^{2}$ ). A cada sub-muestra contenida dentro del marco se le midió la profundidad y peso de la pollinaza para obtener datos sobre la cantidad de pollinaza por galera.

Seguidamente, las 3 sub-muestras por galera se mezclaron para obtener una sola muestra compuesta, que se homogenizó y a la que se le realizó un cuarteo según la metodología descrita por Henríquez y Cabalceta (1999) para muestras de suelo. La muestra compuesta resultante por galera, se dividió a su vez en 4 partes de 500 gramos cada una. Cada sub-muestra de 500 gr se colocó en bolsas de papel debidamente identificadas. Una bolsa se envió al Centro de Investigación en Nutrición Animal (CINA) y otra al Centro de
Investigaciones Agronómicas (CIA) para su análisis en fresco.

Las otras 2 bolsas fueron almacenadas por 60 días, bajo condiciones no controladas, en el laboratorio de anatomía y fisiología de la Escuela de Zootecnia de la Universidad de Costa Rica. La temperatura promedio de la sala fue de $24^{\circ} \mathrm{C}$, la temperatura máxima de $27^{\circ} \mathrm{C}$ y la mínima de $17^{\circ} \mathrm{C}$. La humedad relativa (HR) promedio fue de $59 \%$, con un registro máximo de $75 \%$ y mínimo de $43 \%$. Las muestras se almacenaron en diferentes lotes de acuerdo con la fecha de colecta entre noviembre del 2016 y abril del 2018. El material permaneció en bolsas de papel, sobre mesas de trabajo aisladas. El período de almacenaje se estimó al considerar la experiencia de campo de las personas investigadoras y las consultas realizadas a las personas productoras, intermediarias, distribuidoras de pollinaza, y al doble del tiempo reportado por Roothaert y Matthewman (1992), donde se observó degradación de la pollinaza. Posterior al período de almacenaje, a las muestras se les realizó los mismos análisis que a las muestras frescas. A cada muestra, tanto fresca como almacenada, se le realizaron los siguientes análisis: Humedad a $135^{\circ} \mathrm{C}$ (AOAC 930.15), proteína cruda (AOAC 2001.11), fibra detergente neutra (AOAC 2002.04), fibra detergente ácida (AOAC 973.18), nitrógeno ligado a la fibra detergente neutra (Licitra et al. 1996), nitrógeno ligado a la fibra detergente ácido (Licitra et al. 1996), extracto etéreo (AOAC 920.39), energía digestible, nitrógeno no proteico (Licitra et al. 1996), cenizas (AOAC 942.05), lignina AOAC 973.18 y, Ca y P (CIA-SC09-01-01-P10). Los resultados de los análisis de FDN y FDA se utilizaron para la estimación de energía digestible (Weiss 1998). Los análisis de humedad, PC, FDN, FDA, FDN, FDA, EE,NNP, LDA, Peq, cenizas y el cálculo de $\mathrm{ED}$, se realizaron en el Centro de Investigación en Nutrición Animal (CINA) de la Universidad de Costa Rica. Los análisis de contenido mineral se realizaron en el Centro de Investigaciones Agronómicas (CIA) de la Universidad de Costa Rica.

Se realizó un estudio observacional analítico transversal para comparar variables de interés 
nutricional en muestras de pollinaza (humedad, PC, FDN, FDA, FDN, FDA, EE, NNP, LDA, Peq, cenizas, Ca, P y ED), categorizadas según tipo de alojamiento de las galeras (abiertas vs cerradas), tipo de uso de la cama de la galera (reuso vs no reuso) y efecto de almacenamiento (pollinaza fresca $v s$ pollinaza almacenada). Se utilizó la prueba t de student para muestras independientes con el fin de determinar diferencias estadísticas entre tipos de alojamiento y tipo de uso de la cama de la galera. Mediante la prueba de Wilcoxon se logró investigar las diferencias entre muestras frescas de pollinaza y muestras de pollinaza después de un período de almacenamiento de 2 meses, esto por cuanto los datos no cumplieron el supuesto de normalidad según la prueba de Shapiro-Wilks. Se realizó una prueba de comparación múltiple de Tukey protegida por un análisis de varianza (ANDEVA) para determinar diferencias entre características generales de las galeras asociadas a cada sistema productivo. Se fijó a priori un valor p de 0,05 como umbral para declarar la significancia de las pruebas estadísticas. Para el análisis de datos, se utilizó el software estadístico Infostat ${ }^{\circledR}$ (v. 2017) (Di Rienzo et al. 2017).

\section{RESULTADOS Y DISCUSIÓN}

La pollinaza constituye una buena fuente de proteína y minerales para la alimentación de rumiantes (Arroyo et al. 2003, Arce et al. 2015), pero su contenido nutricional es muy variable (Roothaert y Matthewman 1992). Como se observa en el Cuadro 3, el porcentaje de PC de las muestras estudiadas fue de 21,9\% y similar a lo reportado por Van Ryssen (2001), Olson y Daniel (2005), Bolan et al. (2010), pero menor a lo encontrado por Tobía y Vargas (2000a), Tobía y Vargas (2000b), quienes reportaron valores de $34,9 \%$ en un estudio previo realizado en Costa Rica. Morales et al. (2002) reportan valores de $31,6 \%$ en muestras de pollinaza evaluadas en México. Ortiz et al. (2006) encontraron valores de PC de $11,56 \%$ en pollinaza de cascarilla de arroz. Roothaert y Matthewman (1992) mencionan que los valores de PC en la pollinaza varían entre 17 y $31 \%$.

Cuadro 3. Composición nutricional promedio de la pollinaza fresca, en porcentaje de materia seca.

\begin{tabular}{lccc}
\hline Nutriente & Promedio & D.E. & $\mathrm{n}$ \\
\hline MS(\%) & 70,5 & 7,6 & 26 \\
PC\% & 21,9 & 3,7 & 26 \\
EE\% & 2,6 & 1,0 & 24 \\
Cenizas\% & 21,3 & 2,6 & 24 \\
FC\% & 24,7 & 4,5 & 24 \\
ELN\% & 29,5 & 4,2 & 24 \\
FDN\% & 50,2 & 6,1 & 20 \\
FDA\% & 32,0 & 6,6 & 20 \\
LDA\% & 6,5 & 2,4 & 20 \\
PEq\%* & 0,7 & 0,3 & 16 \\
PEq $(\%$ PC) & 18,8 & 7,0 & 16 \\
NNP\%* & 1,7 & 0,6 & 20 \\
NNP $(\%$ PC)*** & 50,6 & 16,1 & 20 \\
Ca\% & 1,8 & 0,5 & 26 \\
P\% & 1,0 & 0,3 & 26 \\
ED (Mcal.kg-1) & 2,2 & 0,3 & $<$ \\
\hline
\end{tabular}

* Proteína equivalente: Hace referencia solamente a nitrógeno proveniente de urea** Hace referencia a todos los compuestos nitrogenados que no forman parte de las proteínas. D.E: desviación estándar***. Proporción de la proteína cruda que corresponde a NNP. 
El contenido de PC de la pollinaza se ve afectado por diversos factores como el tipo de material de cama. Ortiz et al. (2006) encontraron menores contenidos de PC en pollinazas con cama de cascarilla de arroz, comparadas con camas de bagazo de caña. Otro factor que contribuye al bajo contenido de PC, es el alto contenido de FC, que en el caso de las pollinazas analizadas fue de $24,7 \%$ y se considera elevado de acuerdo con lo descrito por Morales et al. (2002).

El tipo de ave, el tipo de alimento que consumen (Roothaert y Matthewman 1992, Ferguson et al. 1998), el desperdicio de alimento (Leytem et al. 2007), el tipo de instalaciones y equipo (Tasistro et al. 2004) y la cantidad de aves también afectan la composición de la pollinaza (Roothaert y Matthewman 1992). El porcentaje de PC observado es diferente al reportado en estudios previos realizados en Costa Rica (Tobía y Vargas 2000a, Tobía y Vargas 2000b) y se puede atribuir al uso de comederos o sistemas de alimentación más eficientes (comederos automáticos), al uso de alimento peletizado (Abdollahi et al. 2018)"container-title":"Animal Feed Science and Technology","page":"154-165","volume": "237","source":"ScienceDirect","abstract":"Fe ed intake (FI, al uso de cascarilla de arroz, a la mayor cantidad de aves alojadas por $\mathrm{m}^{2}$, al uso de instalaciones cerradas y al uso de dietas para pollo de engorde con menores contenido de proteína cruda (Ferguson et al. 1998). Estas mejoras evitan el desperdicio de alimento por parte de los animales e incrementan el contenido de proteína verdadera de la pollinaza.

Por su contenido de PC, la pollinaza se recomienda como un ingrediente para ser incluido en las dietas de rumiantes, bovinos y ovinos (Tobía y Vargas 2000a, Van Ryssen 2001, Arroyo et al. 2003, Olson y Daniel 2005, Ortiz et al. 2006, Arce et al. 2015). El porcentaje de PC de la pollinaza, observado en este trabajo, es similar a los contenidos de PC reportados por Sánchez et al. (2000), en algunos pastos tropicales (Arachis pintoi, Cynodon nlemfuensis,
Brachiaria dictyoneura, Brachiaria brizantha) en Costa Rica.

Las pollinazas analizadas mostraron un promedio de $1,7 \%$ de NNP, porcentaje menor a lo reportado por Bolson et al. (2010) y por Tobía y Vargas (2000a). Los microorganismos ruminales pueden utilizar las fuentes de NNP como fuente de proteina dietética (Roothaert y Matthewman 1992). Un menor contenido de NNP como porcentaje de la materia seca total, puede ser el resultado de un mejor aprovechamiento del nitrógeno del alimento por parte de las aves que provoca una menor cantidad en las excretas, producto de la mejora en las prácticas de formulación específica de las dietas avícolas (Roothaert y Matthewman 1992, Ferguson et al. 1998). Las dietas actuales se formulan por perfiles ideales de aminoácidos (incluidos sintéticos), y utilizan aditivos como enzimas, promotores de crecimiento, pre y probióticos y ácidos orgánicos que contribuyen en la digestibilidad de las dietas (Nahm 2007, Lu et al. 2017) para pollos.

Un menor contenido de NNP en la pollinaza se podría considerar como un aspecto positivo, si se toma en cuenta que niveles elevados de nitrógeno en la dieta de los rumiantes pueden provocar problemas en la respuesta reproductiva y causar toxicidad (Shalton y Whitter 2006). El NNP en niveles altos de inclusión, puede llegar a tener impactos negativos en el desempeño reproductivo en rumiantes y puede ser tóxico (Staton y Whittier 2006). Un alto consumo de NNP produce una elevada concentración de amoníaco en el rumen y eventualmente un incremento de la concentración de urea en sangre (BUN). El aumento de la urea en sangre provoca disminución del pH uterino e interfiere con la producción de progesterona, lo que crea condiciones uterinas poco favorables para la implantación del feto y desarrollo de la preñez (Laven y Drew 1999, Hammon et al. 2005). Este mismo incremento en la concentración de BUN, ya sea debido a un muy alto consumo de NNP o a una baja disponibilidad de carbohidratos rápidamente fermentables que provean energía para la creación de proteína 
microbiana, puede ocasionar un efecto tóxico y rápidamente letal en el animal (Parish 2018). Se requiere más investigación para conocer los porcentajes máximos de inclusión, que no afecten la salud de los animales.

El contenido de NNP, como porcentaje de la proteína cruda observado, fue de 50,6\%, lo que coincide con lo reportado por Roothaert y Matthewman (1992, Van Ryssen (2001), quienes observaron que la proporción de proteína verdadera y del NNP en la pollinaza varía entre 40 y $50 \%$. Tobía y Vargas (2000b) reportan un 46,9\% de NNP como porcentaje de la proteína cruda.

Las muestras de pollinaza analizadas mostraron un valor promedio de cenizas de 21,3\%, valor que se encuentra dentro del rango recomendado para el uso de pollinaza como alimento y es similar a los reportado por Ortiz et al. (2006), pero mayor a lo reportado por Tobía y Vargas (2000a). Williams et al. (1999) no recomiendan el uso de camas con más de $28 \%$ de cenizas para la alimentación animal. Un contenido alto de cenizas en la pollinaza se asocia con presencia de materiales extraños como plástico y plumas, y a instalaciones sin piso de concreto (Olson y Daniel 2005, Bolson et al. 2010). Altas concentraciones de cenizas pueden diluir la concentración de otros nutrientes como la energía digestible en la pollinaza (Van Ryssen 2001).

La pollinaza se considera un ingrediente deficiente en energía (Van Ryssen 2001). El contenido energético observado $\left(2,2 \mathrm{Mcal} . \mathrm{kg}^{-1}\right)$ en las muestras analizadas fue menor al reportado por Tobía y Vargas (2000a), producto de contenidos menores de PC, EE y mayores de la fracción fibrosa. Poca disponibilidad de energía puede resultar insuficiente para que los microorganismos ruminales utilicen eficientemente el nitrógeno (Van Ryssen 2001), por lo que se recomienda suplementar la pollinaza con fuentes de energía como maíz (Olson y Daniel 2005) o melaza (Van Ryssen 2001).

El valor de las fracciones fibrosas (FDN, FDA, LDA) encontrado fue similar al contenido de muchas fuentes forrajeras de buena calidad
(Sánchez et al. 2000, Arce et al. 2015) de Costa Rica. La mezcla de heces, con los demás contenidos de la cama, pueden diluir el contenido de la fibra en la pollinaza (Ortiz et al. 2006). El porcentaje de FC $(24,7)$ fue similar al encontrado por Olson y Daniel (2005). Ortiz et al. (2006) reportan mayores contenidos de FDN, FDA y LDA.

La pollinaza se considera una buena fuente de calcio y fósforo (Roothaert y Matthewman 1992). El porcentaje de calcio de las pollinazas analizadas fue cercano a lo reportado por Van Ryssen (2001), menor a lo encontrado por Roothaert y Matthewman (1992), Tobía y Vargas (2000a), Bolan et al. 2010 y mayor a lo observado por Morales et al. (2002), Ortiz et al. (2006). $\mathrm{El}$ fósforo de las pollinaza analizadas fue menor a lo reportado por Roothaert y Matthewman (1992), Van Ryssen (2001), Tobía y Vargas (2000a), Morales et al. (2002). La relación calcio: fósforo observada fue de 2:1 comparada con 3:1 observada por Tobía y Vargas (2000a). Es importante conocer la relación calcio:fósforo de las pollinazas, debido que un debalance en esta relación puede predisponer a la presentación de casos de hipocalcemia en vacas gestantes Olson y Daniel 2005.

El porcentaje de materia seca $(70 \%)$ de las pollinazas analizadas fue menor que lo encontrado por Roothaert y Matthewman (1992), Tobía y Vargas (2000a), Morales et al. (2002), Ortiz et al. (2006), (Magdy 2017). Algunas personas autoras mencionan que el valor nutricional de la pollinaza no se ve afectado por el contenido de humedad (Olson y Daniel 2005). Van Ryssen (2001) reporta que la palatabilidad de la pollinaza se puede ver afectada cuando la humedad sobrepasa $200 \mathrm{~g} \cdot \mathrm{kg}^{-1}$.

La pollinaza muestra un atractivo valor nutricional como ingrediente en la alimentación de rumiantes. Sin embargo, su uso está restringido al acatamiento de las normas nacionales e internacionales, que regulan su utilización, basada principalmente en consideraciones sanitarias y no de carácter nutricional. El Reglamento sobre el Manejo y Control de Gallinaza 
y Pollinaza (MAG-S-MINAE 2000), el Decreto $\mathrm{N}^{\circ}$. 29285-MAG-S del 2001, la directriz SENASA-DG-D008-2012: Medidas de precaución para evitar la presencia de la enfermedad Encefalopatía Espongiforme Bovina (EEB), conocida como la "enfermedad de las vacas locas” y el capítulo 11.4 del Código sanitario de las animales terrestres (OIE 2018); regulan los procedimientos para su tratamiento y uso con el fin de evitar la proliferación y diseminación de plagas y enfermedades que afectan tanto animales como humanos.

\section{Efecto del tipo de alojamiento}

No se encontró un efecto significativo por el tipo de alojamiento (galeras convencionales o abiertas y galeras cerradas o tipo túnel) sobre la composición nutricional de la pollinaza $(p>0,05)$ como se muestra en el Cuadro 4. Los contenidos de PC, como de NNP, no presentaron diferencias significativas según el tipo de alojamiento. Esto contrasta con lo observado por otras personas autoras quienes reportan diferencias en el contenido nutricional de la pollinaza debido al sistema de producción (Roothaert y Matthewman 1992, Coufal et al. 2006). Las galeras cerradas tipo túnel alojan una mayor cantidad de aves por $\mathrm{m}^{2}$ y se espera una mayor carga de excretas, $\mathrm{y}$ por tanto, de NNP. La aplicación de un mayor volumen de cama en las galeras cerradas tipo túnel provoca un efecto de dilución de las excretas (Ortiz et al. 2006) que podría explicar este resultado.

Cuadro 4. Composición nutricional de la pollinaza fresca según el tipo de alojamiento de los pollos de engorde.

\begin{tabular}{lccc}
\hline & \multicolumn{2}{c}{ Tipo de alojamiento } & \\
\hline Nutriente (\%MS) & Galera abierta & Galera cerrada & Valor p \\
\hline MS\% & 70,81 & 70,15 & 0,8401 \\
PC\% & 22,39 & 21,40 & 0,5365 \\
NNP\% & 1,45 & 1,85 & 0,3909 \\
NNP-\%PC & 37,76 & 56,15 & 0,0971 \\
PEq\% & 0,67 & 0,69 & 0,9006 \\
PEq - \%PC & 19,19 & 17,88 & 0,7706 \\
EE\% & 2,68 & 2,48 & 0,6560 \\
FC\% & 24,20 & 25,34 & 0,5585 \\
ELN\% & 29,49 & 29,41 & 0,9630 \\
Cen\% & 21,24 & 21,43 & 0,8616 \\
FDN\% & 50,16 & 50,17 & 0,9990 \\
FDA\% & 32,24 & 31,79 & 0,8957 \\
LDA\% & 6,13 & 6,74 & 0,6255 \\
Ca\% & 1,79 & 1,82 & 0,8523 \\
P\% & 1,09 & 0,96 & 0,3143 \\
E.dig (Mcal.kg-1) & 2,15 & 2,16 & 0,9452 \\
\hline
\end{tabular}




\section{Efecto de la reutilización de la cama}

La pollinaza de galeras con camas reutilizadas, en ciclos subsiguientes de producción, mostró diferencias significativas $(\mathrm{p}<0,05)$ en el contenido de proteína cruda, así como en la fracción fibrosa y en el porcentaje de calcio y de fósforo como se observa en el Cuadro 5.

Cuadro 5. Composición nutricional de la pollinaza fresca según el uso de la cama de los pollos de engorde.

\begin{tabular}{lccc}
\hline & \multicolumn{3}{c}{ Uso Cama } \\
\hline Nutriente (\%MS) & Primer Uso & Reutilizada & Valor p \\
\hline MS\% & 71,33 & 68,92 & 0,4835 \\
PC\% & 20,44 & 24,69 & 0,0061 \\
NNP & 1,70 & 1,83 & 0,8204 \\
NNP-\%PC & 51,87 & 45,66 & 0,6520 \\
PEq\% & 0,57 & 0,79 & 0,1304 \\
PEq $\%$ PC & 17,81 & 19,93 & 0,6079 \\
EE\% & 2,59 & 2,58 & 0,9962 \\
FC\% & 26,15 & 22,10 & 0,0384 \\
ELN\% & 30,02 & 28,39 & 0,3844 \\
Cen\% & 20,80 & 22,33 & 0,1822 \\
FDN\% & 51,77 & 44,96 & 0,0474 \\
FDA\% & 33,56 & 26,84 & 0,0741 \\
LDA\% & 6,62 & 6,05 & 0,6887 \\
Ca\% & 1,64 & 2,11 & 0,0192 \\
P\% & 0,92 & 1,23 & 0,0156 \\
ED $($ Mcal.kg-1) & 2,16 & 2,15 & 0,9578 \\
\hline
\end{tabular}

En el caso de la PC, en las galeras con cama reutilizada, se obtuvo concentraciones mayores que en galeras con cama de un solo uso, similar a lo observado por Ortiz et al. (2006). De acuerdo con Lu et al. (2017) los compuestos nitrogenados simples que componen el NNP poseen una alta volatilidad, por lo que el volteo y tratamiento de la cama que se reutiliza, previo al siguiente ciclo, podría ser la causa de las diferencias en los contenidos de PC y que los niveles de NNP no cambien con la reutilización.
La fracción fibrosa mostró diferencias significativas, con menores concentraciones para la cama reutilizada, resultados semejantes fueron observados por Van Ryssen (2001). La fracción proteica total podría aumentar por adición de alimento desperdiciado y podría alterar la relación proteína/fibra de la pollinaza. También se reporta que altos contenidos de FC afectan la PC (Morales et al. 2002).

El picoteo de la cascarilla de arroz de la cama, por parte de las aves, también podría 
causar disminución de la fracción digestible de la fibra de la pollinaza. En sistemas de producción avícola con ciclos mucho más largos, como los de gallinas ponedoras en sistemas de piso, al final de proceso la cascarilla de arroz es prácticamente no identificable en la cama.

Aunque se observaron diferencias significativas en el contenido de calcio y fósforo, con mayores concentraciones en las galeras con camas reutilizadas, la relación calcio/fósforo se mantiene cercana a 1,8 en ambos casos. Souza et al. (2012) mencionan que el contenido de $\mathrm{P}$ en la pollinaza puede aumentar cuando se agrega material de cama y este sufre degradación.

\section{Efecto del almacenamiento}

Se observó disminución en el porcentaje de humedad en la pollinaza almacenada (Cuadro 6), similar a lo reportado por Ortiz et al. (2006). La fracción proteica, tanto la PC total como el NNP fueron significativamente menores $(\mathrm{p}<0,05)$ en las muestras almacenadas, tal como lo reportan Roothaert y Matthewman (1992). Esto se podría atribuir en parte a la volatilidad de los compuestos nitrogenados simples, apoyado por el aumento en la concentración de NNP como porcentaje de la PC total en la pollinaza fresca (Chambliss 2018).

Cuadro 6. Composición nutricional de la pollinaza almacenada.

\begin{tabular}{lccll}
\hline Nutriente & Almacenada & Fresca & p-valor & $\mathrm{n}$ \\
\hline MS\% & 85,15 & 71,70 & $<0,0001$ & 17 \\
PC\% & 20,65 & 22,14 & 0,0160 & 17 \\
NNP\% & 1,24 & 1,77 & 0,0420 & 7 \\
NNP-\%PC & 37,18 & 48,83 & 0,0264 & 7 \\
EE\% & 1,56 & 2,64 & $<0,0001$ & 17 \\
FC\% & 23,97 & 24,54 & $>0,9999$ & 17 \\
ELN\% & 32,63 & 29,65 & $<0,0001$ & 17 \\
Cen\% & 21,18 & 21,07 & 0,6116 & 17 \\
FDN\% & 52,60 & 49,99 & 0,0728 & 11 \\
FDA\% & 32,46 & 32,05 & 0,4984 & 11 \\
LDA\% & 6,08 & 6,69 & $>0,9999$ & 11 \\
P\% & 1,03 & 0,98 & 0,2976 & 16 \\
Ca\% & 1,91 & 1,80 & 0,1624 & 16 \\
E.dig (Mcal.kg-1) & 2,12 & 2,15 & 0,6744 & 11 \\
\hline
\end{tabular}

El extracto etéreo también disminuyó en la pollinaza almacenada, así como el extracto libre de nitrógeno $(\mathrm{p}<0,05)$. Estas diferencias observadas pueden ser atribuibles a la descomposición y degradación de los compuestos (Tasistro et al. 2004), así como consecuencia de la presencia de microorganismos que degradan el nitrógeno y otros compuestos en la pollinaza (Atkinson et al. 1996, Gómez 2006). El efecto en la disminución de la humedad, producto del almacenamiento, podría ocasionar que el contenido por unidad de peso de estos nutrientes sea mayor en la pollinaza 
almacenada (Chambliss 2018). La fracción fibrosa y los minerales no mostraron diferencias significativas ( $p>0,05)$, debido a que ambos componentes nutricionales son muy estables (Atkinson et al. 1996) a las condiciones ambientales a las que fue sometida la pollinaza almacenada en este experimento.

\section{Características del alojamiento y de la cama}

En galeras cerradas o tipo túnel, con cama reutilizada, se obtuvo mayor cantidad de pollinaza expresada en $\mathrm{kg}$ por $\mathrm{m}^{2}$ que en las galeras abiertas y cerradas sin reutilizar, debido a la mayor cantidad de aves alojadas, a la presencia de humedad y a la mayor cantidad de cama, tal como se observa en el Cuadro 7. Cuando se reutilizó la cama, en galeras abiertas o convencionales se obtuvo mayor cantidad de pollinaza por pollo que en las galeras cerradas sin reutilizar. La cantidad de pollinaza por pollo en los sistemas abiertos fue mayor que lo observado por Tobía y Vargas (2000a), esto debido a que las galeras analizadas en este trabajo tenían una mayor cantidad de animales, así como de pollinaza, por metro cuadrado.

Cuadro 7. Características de alojamiento de los pollos de engorde en sistemas de producción convencionales o abiertos y cerrados tipo túnel con cama reutilizada y de un solo uso.

\begin{tabular}{|c|c|c|c|c|c|c|}
\hline & \multicolumn{2}{|c|}{$\begin{array}{c}\text { Galeras convencionales o } \\
\text { abiertas }\end{array}$} & \multicolumn{2}{|c|}{$\begin{array}{c}\text { Galeras cerradas o tipo } \\
\text { túnel }\end{array}$} & \multicolumn{2}{|l|}{$\begin{array}{l}\text { Galeras } \\
\text { abiertas }\end{array}$} \\
\hline & $\begin{array}{l}\text { cama sin } \\
\text { reutlizar }\end{array}$ & $\begin{array}{l}\text { cama } \\
\text { reutilizada }\end{array}$ & $\begin{array}{l}\text { cama sin } \\
\text { reutilizar }\end{array}$ & $\begin{array}{c}\text { cama } \\
\text { reutilizada }\end{array}$ & $\begin{array}{c}\text { Tobía y } \\
\text { Vargas } 2000\end{array}$ & p-valor \\
\hline Densidad:pollos por $\mathrm{m}^{2}$ & 12,52 & 12,52 & 16,9 & 16,9 & $11,9 \pm 1,7$ & \\
\hline Peso de la pollinaza $\mathrm{kg}$ por $\mathrm{m}^{2}$ & $19,8 \pm 1,2 \mathrm{a}$ & $27,9 \pm 5,1 \mathrm{ab}$ & $20,7 \pm 4,0 \mathrm{a}$ & $34,3 \pm 8,5 b$ & $15,2 \pm 2,6$ & 0,0127 \\
\hline Profundidad de la pollinaza $\mathrm{cm}$ & $4,8 \pm 1,0 \mathrm{a}$ & $8,5 \pm 2,5 b$ & $5,1 \pm 0,9 \mathrm{a}$ & $6,9 \pm 0,7 \mathrm{ab}$ & $\mathrm{Nd}$ & 0,0010 \\
\hline Producción de pollinaza por (pollo)* & $1,48 \mathrm{ab}$ & $2,25 b$ & $1,33 \mathrm{a}$ & $1,90 \mathrm{ab}$ & 1,28 & 0,0177 \\
\hline Tipo de piso & $\begin{array}{l}\text { Concreto } \\
100 \%\end{array}$ & $\begin{array}{l}\text { Concreto } \\
100 \%\end{array}$ & $\begin{array}{l}\text { Concreto } \\
100 \%\end{array}$ & $\begin{array}{c}\text { Concreto } \\
100 \%\end{array}$ & $\begin{array}{l}34 \% \text { piso de } \\
\text { concreto }\end{array}$ & \\
\hline $\mathrm{N}^{\circ}$ de ciclos de producción por año & 6,8 & 6,8 & 6,8 & 6,8 & 6,0 & \\
\hline Tipo de cama & $\begin{array}{c}\text { Cascarilla } \\
\text { de arroz } \\
100 \%\end{array}$ & $\begin{array}{c}\text { Cascarilla } \\
\text { de arroz } \\
100 \%\end{array}$ & $\begin{array}{c}\text { Cascarilla } \\
\text { de arroz } \\
100 \%\end{array}$ & $\begin{array}{c}\text { Cascarilla } \\
\text { de arroz } \\
100 \%\end{array}$ & $\begin{array}{c}\text { Cascarilla de } \\
\text { arroz } 80 \% \\
\text { y viruta de } \\
\text { madera } 20 \%\end{array}$ & \\
\hline
\end{tabular}

Los datos se presentan con una desviación estándar.

*Peso de la pollinaza entre densidad de pollos.

Los datos mostraron más ciclos productivos por año, el material del piso de las galeras fue el concreto y el $100 \%$ de las camas fueron de cascarilla de arroz en comparación con lo observado por Tobía y Vargas (2000a).

\section{CONCLUSIONES}

En esta investigación se observó que la composición nutricional de la pollinaza puede cambiar con las prácticas de manejo de las 
granjas avícolas como la reutilización de la cama y el tiempo de almacenamiento del material. Por esta razón, la actualización periódica de la composición nutricional de este ingrediente y de todos los componentes de una dieta son básicos para un adecuado balance nutricional y para garantizar la salud de los animales. La composición nutricional, de la pollinaza observada, permite considerarla como una opción o ingrediente en la alimentación de bovinos en general y especialmente en condiciones de desastres naturales, sequía y poca disposición de forraje y así como una alternativa para la disposición de los residuos de la industria avícola, siempre y cuando sean consideradas las disposiciones del Servicio Nacional de Salud Animal (SENASA) y de otras instituciones gubernamentales e internacionales que regulan su uso y manejo.

\section{AGRADECIMIENTOS}

Las personas autoras expresan su agradecimiento a los avicultores y comercializadores que colaboraron en el desarrollo de este proyecto.

\section{LITERATURA CITADA}

Abdollahi, M; Zaefarian, F; Ravindran, V. 2018. Feed intake response of broilers: Impact of feed processing. Animal Feed Science and Technology 237:154-165.

Arce, J; Rojas, A; Poore, M. 2015. Efecto de la adición de pollinaza sobre las características nutricionales y fermentativas del ensilado de subproductos agroindustriales de yuca (Manihot esculenta). Agronomía Costarricense 39(1):131-140.

Arroyo, C; Rojas Bourrillón, A; Rosales, R. 2003. Urea o pollinaza como suplemento proteico para toretes consumiendo ensilaje de pulpa de pejibaye. Agronomía Costarricense 27(2):69-73.

Atkinson, C; Jones, D; Gauthier, J. 1996. Biodegradability and microbial activities during composting of poultry litter. Poultry Science 75(5):608-617.

Bolan, NS; Szogi, AA; Chuasavathi, T; Seshadri, B; Rothrock Jr, MJ; Penneerselvam, P. 2010. Uses and management of poultry litter. World's Poultry Science Journal 66(04):673-698.

Cayci, G; Temiz, C; Sözudogru, S. 2017. The effects of fresh and composted chicken manures on some soil characteristics. Communications in Soil Science and Plant Analysis 48(1):1-11.
Chambliss, A. 2018. Factors Affecting the Nutrient Content and Composition of Poultry Litter (en línea). Extension Alabama and Auburn Universities. Consultado mar. 2019. Disponible en http://www. sites.aces.edu/group/crops/blog/List/Post/Post. aspx?ID=234

Coufal, C; Chavez, C; Niemeyer, P; Carey, J. 2006. Measurement of broiler litter production rates and nutrient content using recycled litter. Poultry Science 85(3):398-403.

Decreto No. 29285-MAG-S. 2001. Prohibe el Uso de cualquier Componente o Material Específico Proveniente de Rumiantes, sean Nacionales o Importadas en la Alimentación de Rumiantes. Diario oficial La Gaceta. Costa Rica. 13 set. 2 p.

Directriz SENASA- DG-D008. 2012. Medidas de precaución para evitar la presencia de la enfermedad Encefalopatía Espongiforme Bovina (EEB), conocida como la "enfermedad de las vacas locas". Diario oficial La Gaceta. Costa Rica. 6 p.

Di Rienzo, JA; Casanoves, F; Balzarini, MG; Gonzalez, L; Tablada, M; Robledo, CW. 2017. InfoStat versión 2017. Grupo InfoStat, FCA (en línea, programa informático). Córdoba, Argentina, Universidad Nacional de Córdoba. Consultado 04 may. 2018. Disponible en http://www.infostat.com.ar

Ferguson, N; Gates, R; Taraba, J; Cantor, A; Pescatore, A; Straw, ML; Burnham, D. 1998. The effect of dietary protein and phosphorus on ammonia concentration and litter composition in broilers. Poultry Science 77(8):1085-1093.

Galvis, R; Correa, H; Barrientos, S; Muñoz, Y. 2011. Efecto de los niveles crecientes de nitrógeno no proteico dietario en vacas lactantes sobre las concentraciones de metabolitos nitrogenados en orina, sangre y leche. Revista Facultad Nacional de Agronomía de Medellín 64 (2):6191-6198.

Garriz, M; López, A. 2002. Suplementación con nitrógeno no proteico en rumiantes. Monografía Final del Curso: "Nutrición en la Intensificación". Cátedra de Nutrición y Alimentación Animal de la Facultad de Veterinaria de la Universidad de Buenos Aires (en línea). Buenos Aires, Argentina. Consultado $30 \mathrm{feb}$. 2018. Disponible en http://produccionanimal.com. ar/informacion_tecnica/suplementacionproteica_y_ con_nitrogeno_no_proteico/07suplementacion_ con_nitrogeno.pdf

Glatz, P; Pym, R. 2019. Poultry housing and management in developing countries (en línea). Poultry development review. FAO. Organización de las Naciones Unidas para la Alimentación y la Agricultura. Consultado 6 mar. Disponible en https://www.google.com/search? $\mathrm{q}=$ Poultry+housing+and+management+in+developi ng+countries\&rlz=1C5CHFA_enCR776CR781\&oq $=$ Poultry+housing+and+management+in+developin 
g+countries\&aqs=chrome..69i57.1487j0j8\&sourceid $=$ chrome $\&$ ie $=$ UTF- 8

Gómez, D. 2006. Evaluación y caracterización del proceso de biodegradación de pollinaza en camas usando microorganismos. Trabajo presentado como requisito parcial para optar por el título de: Ingeniería Química. Universidad Industrial de Santander, Bucaramanga, Colombia.

Henríquez, C; Cabalceta, G. 1999. Guía práctica para el estudio introductorio de los suelos con un enfoque agrícola. San José, Costa Rica. Asociación Costarricense de la Ciencia del Suelo. 112 p.

Hammon, D; Holyoak, G; Dhiman, T. 2005. Association between blood plasma urea nitrogen levels and reproductive fluid urea nitrogen and ammonia concentrations in early lactation dairy cows. Animal Reproduction Science 86 (3-4):195-204.

INEC (Instituto Nacional de Estadística y Censos). 2014. VI Censo Nacional Agropecuario. Características de las fincas y de las personas productoras.1 ed. San José, Costa Rica. 178 p.

INEC (Instituto Nacional de Estadsitica y Censos). 2017. Una visión del sector agropecuario basado en el CENAGRO 2014.1 ed. San José, Costa Rica. 588 p.

Laven, R; Drew, S. 1999. Dietary protein and the reproductive performance of cows. Veterinary Record 145:687695.

Leytem, A; Plumstead, P; Maguire, R; Kwanyuen, P; Brake, J. 2007. What aspect of dietary modification in broilers controls litter water-soluble phosphorus: dietary phosphorus, phytase, or calcium? Journal of Environmental Quality 36(2):453-463.

Licitra, G; Hernandez, T; Van Soest, P. 1996. Standardization of procedures for nitrogen fractionation of ruminant feeds. Animal Feed Science and Technology 57(4):347-358.

Lu, L; Liao, X; Luo, X. 2017. Nutritional strategies for reducing nitrogen, phosphorus and trace mineral excretions of livestock and poultry. Journal of Integrative Agriculture 16(12):2815-2833.

Magdy, S. 2017. More nutrients and more hazards when using poultry litter in plant and/or animal nutrition. Nutrition and Food Science International Journal 4(2). 555635. Doi: 10.19080/NFSIJ.2017.04.555635

Mesa,D; Muniz,E; Souza, A; Geffroy,B.2017.Broiler-Housing Conditions Affect the Performance. Brazilian Journal of Poultry Science 19(2):263-272.

Morales, H; Gutiérrez, E; Bernal, H. 2002. El uso de cama de pollo de buena calidad mejora la productividad de bovinos en crecimiento en engorda intensiva. Técnica Pecuária En México 40(1):1-15.

Nahm, K. 2007. Feed formulations to reduce N excretion and ammonia emission from poultry manure. Bioresource Technology 98(12):2282-2300.

OIE (Organización Mundial de Sanidad Animal). 2018. Encefalopatía Espongiforme Bovina. Capítulo 11.4.
Código sanitario de los animales terrestres (en línea). Consultado 19 feb. Disponible en:http://oie. intint/fileadmin/Home/esp/Health_standards/tahc/ current/chapitre_bse.pdf

Olson, K; Daniel, J. 2005. Feeding Poultry Litter to Beef Cattle (en línea). Extension Publications (MU). Consultado feb. 2019. Disponible en https://mospace. umsystem.edu/xmlui/handle/10355/3354

Ortiz, A; Elias, A; Valdivie, M; Gonzalez, R. 2006. Poultry litter, a way of increasing the nutritive value of highly fibrous materials. Cuban Journal of Agricultural Science 40(1):55-60.

Paganini, F; Mendes, A; Naas, I; Macar, M. 2004. Manejo da cama. Produção de Frangos de Corte. Facta Brasil 1(1):108-115.

Parish, JA. 2018. Protein in Beef Cattle Diets (en línea). Mississippi State University Extension. USA. Consultado 11 may. 2018. Disponible en http:// extension.msstate.edu/sites/default/files/ publications/publications/p2528_0.pdf

PNSA (Programa Nacional de Salud Aviar). 2019. Servicio Nacional de Salud Animal. Ministerio de Agricultura y Ganadería (en línea). Consultado 4 mar. Disponible en http://www.senasa.go.cr/informacion/centro-deinformacion/informacion/sgc/pnsa

Reglamento No 29145 MAG-S-MINAE, 2000. Reglamento Sobre el Manejo y Control de Gallinaza y Pollinaza. Diario oficial La Gaceta. Costa Rica. 18 dic.

Roothaert, R; Matthewman, R. 1992. Poultry wastes as foods for ruminants and associated aspects of animal welfare - Review. Asian-Australasian Journal of Animal Sciences 5(4):593-600.

Sánchez, J; Villarreal, M; Soto, H. 2000. Caracterización nutricional de los componentes forrajeros de cuatro asociaciones gramíneas Arachis pintoi. Nutrición Animal Tropical 6(1):1-22.

Souza, C; Ghosh, A; Silva, I; Alvarenga, E; Novais, R; Jesus, G. 2012. Phosphorus transformation in poultry litter and litter-treated Oxisol of Brazil assessed by 31P-NMR and wet chemical fractionation. Revista Brasileira de Ciencia Do Sol 36(5):1516-1527.

Stanton, T; Whittier, J. 2006. Urea and NPN for cattle and sheep (en línea). In Colorado State University Cooperative Extension - Agriculture, Document, 1.608. 3 p. Consultado 5 mar. Disponible en https:// extension.colostate.edu/topic-areas/agriculture/ureaand-npn-for-cattle-and-sheep-1-608-2/

Tasistro, A; Kissel, D; Bush, P. 2004. Spatial variability of broiler composition in a chicken house. Journal of Applied Poultry Research 13:29-43.

Tobía, C; Vargas, E. 2000a. Evaluación de las Excretas de Pollo de Engorde en la Alimentación Animal I: Disponibilidad Y Composición Química. Agronomía Costarricense 24(1):47-53.

Tobía, C; Vargas, E. 2000b. Evaluación de las Excretas de Pollo de Engorde en la Alimentación Animal II: 
Fraccionamiento de Componentes Nitrogenados y Contenido de Energía. Agronomía Costarricense 24(1):55-62.

Tokarnia, C; Döbereiner, J; Peixoto, P; Moraes, S. 2000. Outbreak of copper poisoning in cattle fed poultry litter. Veterinary and Human Toxicology 42(2):92-95.

Van Ryssen, J. 2001. Poultry litter as a feedstuff for ruminants: A South African scene. South African Journal of Animal Science 2:1-8.

Vejarano, M; Alba; M; Reyna, P; Casas,E. 2008.Comparación productiva de pollos de carne criados en camas nuevas vs. cama reutilizada por cinco campañas. Revista de Investigaciones Veterinarias del Perú 19 (2):126-133.

Weiss W. 1998. Energy prediction equations for ruminant feeds. Department of Animal Sciences. Ohio State University, Wooster. 9 p.

Williams, C; Barker, J; Sims, J. 1999. Management and Utilization of Poultry Wastes. Reviews of Environmental Contamination and Toxicology: Continuation of Residue Reviews. New York, EEUU. Springer New York. p. 105-157. 
